\title{
Prevertebral space effusion caused by the breaking of swollen lymphonodi retropharynici in nasopharyngeal carcinoma: A case report
}

\author{
LIAN-HUA LIU ${ }^{1}$ and XIAO-FENG YANG ${ }^{2}$ \\ ${ }^{1}$ Department of Radiotherapy, The Linyi Cancer Hospital, Linyi, Shandong 276000; \\ ${ }^{2}$ Department of Emergency, The Linyi People's Hospital, Linyi, Shandong 276001, P.R. China \\ Received February 14, 2015; Accepted January 18, 2016
}

DOI: $10.3892 / \mathrm{ol} .2016 .4473$

\begin{abstract}
A 20-year-old male presented to hospital with blood in the mucus, which had occurred for 6 months, and was diagnosed with nasopharyngeal carcinoma (NPC). Due to passive smoking, the patient developed a constant and violent cough, pain behind the right ear and swelling of the retropharynx. The results of a computed tomography scan, which was performed during re-examination of the patient, revealed that the right lymphonodi retropharynici had decreased in size and the hypodense shadow of the prevertebral space had become clear. Therefore, the retropharyngeal pressure was suspected to be increased due to the violent cough. Consequently, the increased pressure may have caused the swollen lymph nodes on the right side of the pharynx to break, which allowed the necrotic liquid to seep into the prevertebral space and result in effusion. Subsequent to 5 days of treatment with methylprednisolone (40 mg ivgtt qd, d1-5), the results from the cone bean computed tomography examination, which had been performed prior to radiotherapy, indicated that the retropharyngeal swelling had improved and the prevertebral space had returned to normal. To the best of our knowledge, the present study is the first reported case of effusion in the prevertebral space caused by the breaking of swollen lymphonodi retropharynici in NPC. The present study acts as a reminder of the possible delays in the treatments for head and neck tumor patients that demonstrate swollen retropharyngeal lymph nodes due to the occurrence of effusion in the prevertebral space.
\end{abstract}

\section{Introduction}

Nasopharyngeal carcinoma (NPC) is one of the most common malignant head and neck tumors. Due to the fast metastasis

Correspondence to: Professor Xiao-Feng Yang, Department of Emergency, The Linyi People's Hospital, 7 Jiefang Road, Linyi, Shandong 276001, P.R. China

E-mail: bsgxyx@163.com

Key words: nasopharyngeal carcinoma, prevertebral space, effusion to the lymph nodes, swelling is frequently indicated in the neck, retropharynx and parapharynx (1). The survival rate of terminal stage patients ranges between 50-60\%, and patients with NPC possess a relatively poor prognosis (2). The prevertebral space is located at the retropharynx or behind the esophagus, with the prevertebral fascia in front. The prevertebral fascia is a thick and dense tissue that extends between the deep surface of the sternocleidomastoid muscle, attached to the skull base, and the thoracic mediastinum, of which one side attaches to the cervical transverse position in front of the vertebrae and the prevertebral muscle and the rear side attaches to the cervical acantha. In addition, retropharyngeal lymph nodes are located in front of the prevertebral fascia (3). The present study reports a rare clinical case, in which effusion of the prevertebral space has been caused by the breaking of swollen lymphonodi retropharynici.

\section{Case report}

A 20-year-old male patient was admitted to the Department of Radiotherapy, The Linyi Cancer Hospital (Linyi, China) on 16 January 2015 due to nasal obstruction and associated epistaxis for a period of 6 months. A magnetic resonance imaging (MRI) scan of the nasopharynx was completed the same day, and the results revealed abnormal masses on the posterior wall of the nasopharynx, the disappearance of the fossa and lateral pharyngeal wall on each side, multiple swollen lymph nodes on each side of the parapharynx, the retropharynx and the neck, and a normal prevertebral space (Fig. 1A). A pathological biopsy was performed on the nasopharyngeal mass, and consequently the patient was diagnosed with NPC.

The patient coughed violently and constantly due to passive smoking, and on 18 January 2015, the coughing was accompanied by a sudden pain behind the right ear and significant feeling of swelling on the retropharynx wall. The patient was administered anti-inflammatory treatments under the consideration of acute mumps. On the following day, a localized computed tomography (CT) scan revealed that the lymph nodes on the right side had decreased in size. This finding was accompanied by an evident hypodense shadow at the prevertebral space, which had an anteroposterior diameter 

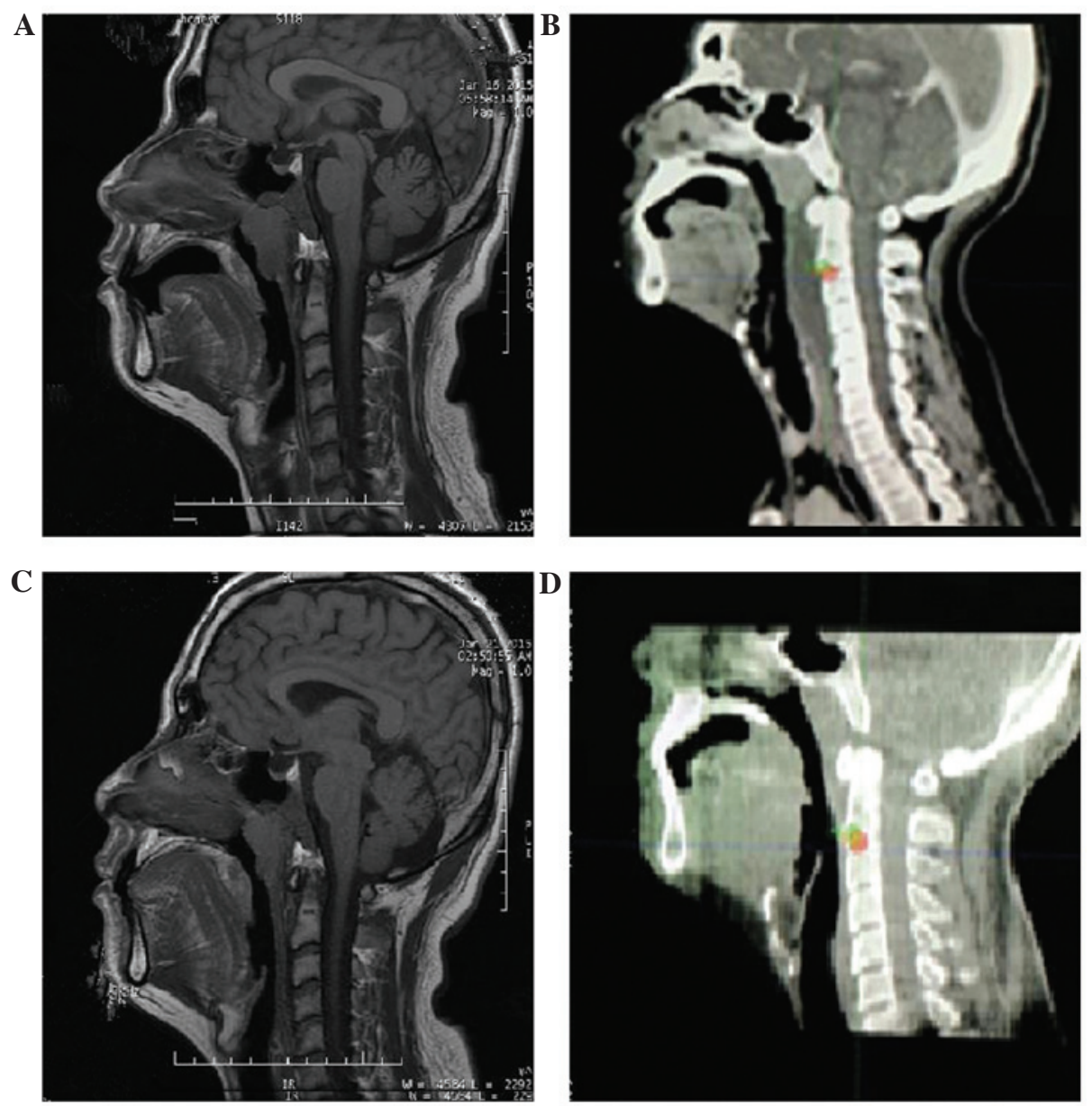

Figure 1. (A) MRI scan revealing abnormal masses on the posterior wall of the nasopharynx and normal prevertebral space. (B) Computed tomography scan revealing a low density shadow (diameter, $2 \mathrm{~cm}$ ) in the prevertebral space. (C) MRI scan revealing that the low density shadow in the prevertebral space had evidently decreased in size (diameter, $1 \mathrm{~cm}$ ). (D) Cone bean computed tomography identification revealing that the prevertebral space had returned to normal. MRI, magnetic resonance imaging.

of $2 \mathrm{~cm}$ (Fig. 1B). The violent cough was considered to have increased the pressure at the retropharynx wall, which then led to the breaking of the swollen lymph nodes in the right pharynx. The broken lymph nodes allowed necrotic liquid to seep into the prevertebral space and cause effusion; therefore, the patient was administered $40 \mathrm{mg}$ methylprednisolone via an intravenous drip. Feedback from the patient subsequent to treatment indicated that the pain on the right parotid gland and the swelling at the retropharynx wall were notably relieved. MRI, which was performed in the re-examination on 21 January 2015, revealed that the prevertebral space was unusually filled. However in comparison with the localized CT scan that was performed 2 days previously, the size of the lesion had evidently decreased to $\sim 1 \mathrm{~cm}$ in diameter (Fig. 1C). Subsequent to continuous treatment with methylprednisolone (ivgtt qd, d1-5), on 26 January 2015, the results of the cone bean computed tomography identification, which was performed prior to the administration of radiotherapy, indicated that the swelling on the retropharynx wall had improved and the prevertebral space had returned to normal (Fig. 1D). In addition, the patient confirmed that the feeling of swelling on the right parotid gland and the retropharynx wall had gone. At present, the patient is undergoing radiotherapy (total dose, $70 \mathrm{~Gy} ; 35$ fractions of 2 Gy over 6 weeks).

\section{Discussion}

Effusion in the prevertebral space is generally considered to be associated with the stimulation of local chronic inflammation. Common causes of effusion in the prevertebral space include odontogenic and pharyngeal infections, sialadenitis, nasosinusitis, wounds and foreign matter on the upper respiratory and upper gastrointestinal tracts, lymphadenitis colli, and fistulae and cysts of the neck (4). Of all the head and neck tumors, NPC is the type that may invade the prevertebral space and cause effusion. In accordance with the study conducted by Liao et al (5), the MRI and CT scans estimated that the occurring rates of the prevertebral muscle in the prevertebral space being attacked are 36.0 and $18.4 \%$, respectively $(\mathrm{P}<0.001)$. In the study conducted by Zhou et al (6), patients with NPC were treated using intensity modulated radiation therapy (IMR), and it was identified that patients with an invaded prevertebral space had dramatically decreased overall survival, distant metastasis-free survival and local recurrence-free survival rates compared with patients with no invaded prevertebral space (6). Therefore, T4 staging is recommended for invasion into the prevertebral space in NPC. For the patient in the present study, the prevertebral space appeared normal in the nasopharyngeal MRI, so the invasion of the prevertebral 
space has been ruled out as the cause of effusion. Following a comprehensive analysis of the effusion, necrosis may have occurred due to interior ischemia of the swollen retropharyngeal lymph nodes. The tissue structures became loose due to the stress on the pharynx wall, produced by the violent cough, which caused the lymph nodes to break. Subsequently, the necrotic fluid seeped into the prevertebral space through the prevertebral fascia and caused effusion. The treatments used were anti-inflammatory and promoted the absorption of methylprednisolone, and were accompanied by appropriate IMR of the target region clinical target volume 1 (CTV1), including the prevertebral fascia and the prevertebral space.

The prevertebral space is located next to the retropharyngeal space, linking to the skull base at the top, the orpharynx at the bottom and the parapharyngeal space at the sides. Therefore, the sclerotin of the skull base and the oral pharynx and parapharyngeal space may be easily affected by effusion. However, as the rear of the prevertebral space is close to the brainstem and the medulla spinalis, the fluid may often cause stress and necrosis in these vital organs. In addition, implantation metastasis and blood metastasis are highly possible results of the fluid released from the broken swollen lymph nodes, which is provided with a T4 staging. The prevertebral fascia and prevertebral space are inclusive in the CTV1 of the radiotherapy target region, but this requires validation from additional clinical studies with a large number of patients. Therefore, for patients with malignant head and neck tumors with swollen lymph nodes on the retropharynx and parapharynx, the clinical symptoms include sudden swelling and pain the behind ears and on the retropharynx. Treatments may be actively administered in order to prevent the occurrence of prevertebral effusion caused by lymph node breakage.

\section{References}

1. Wu TT, Chen C, Chen SM, Xu Y, Wang Y, Chen Z, Wang F, Xiao BK and Tao ZZ: Nuclear translocation of telomerase reverse transcriptase is a critical process in lymphatic metastasis of nasopharyngeal carcinoma. Oncol Lett 9: 265-269, 2015.

2. Wong EY, Wong SC, Chan CM, Lam EK, Ho LY, Lau CP, Au TC, Chan AK, Tsang CM, Tsao SW, et al: TP53-induced glycolysis and apoptosis regulator promotes proliferation and invasiveness of nasopharyngeal carcinoma cells. Oncol Lett 9: 569-574, 2015.

3. Mills MK and Shah LM: Imaging of the perivertebral space. Radiol Clin North Am 53: 163-180, 2015.

4. Regueiro Villarín S, Vázquez Barro JC and Herranz González-Botas J: Deep neck infections: Etiology, bacteriology and treatment. Acta Otorrinolaringol Esp 57: 342-348, 2006 (In Spanish).

5. Liao XB, Mao YP, Liu LZ, Tang LL, Sun Y, Wang Y, Lin AH, Cui CY, Li L and Ma J: How does magnetic resonance imaging influence staging according to AJCC staging system for nasopharyngeal carcinoma compared with computed tomography? Int J Radiat Oncol Biol Phys 72: 1368-1377, 2008.

6. Zhou GQ, Mao YP, Chen L, Li WF, Liu LZ, Sun Y, Chen Y, Tian L, Lin AH, Li L and Ma J: Prognostic value of prevertebral space involvement in nasopharyngeal carcinoma based on intensity-modulated radiotherapy. Int J Radiat Oncol Biol Phys 82: 1090-1097, 2012. 